\title{
The HERMES solar atlas and the spectroscopic analysis of the seismic solar analogue KIC $3241581^{\star, \star \star}$
}

\author{
P. G. Beck ${ }^{1}$, C. Allende Prieto ${ }^{2,3}$, T. Van Reeth ${ }^{4}$, A. Tkachenko ${ }^{4}$, G. Raskin ${ }^{4}$, \\ H. van Winckel ${ }^{4}$, J.-D. do Nascimento Jr. ${ }^{5,6}$, D. Salabert ${ }^{1}$, E. Corsaro ${ }^{1,7,3}$, and R. A. García ${ }^{1}$ \\ ${ }^{1}$ Laboratoire AIM, CEA/DSM CNRS - Univ. Paris Diderot, IRFU/SAp, Centre de Saclay, 91191 Gif-sur-Yvette Cedex, France \\ e-mail: paul.beck@cea.fr \\ 2 Instituto de Astrofísica de Canarias, 38200 La Laguna, Tenerife, Spain \\ 3 Departamento de Astrofísica, Universidad de La Laguna, 38206 La Laguna, Tenerife, Spain \\ 4 Instituut voor Sterrenkunde, KU Leuven, 3001 Leuven, Belgium \\ 5 Harvard-Smithsonian Center for Astrophysics, Cambridge, MA 02138, USA \\ 6 Departamento de Física Teórica e Experimental, Universidade Federal do Rio RJ 21941-901, Brazil \\ 7 Instituto de Astrofísica de Canarias, 38205 La Laguna, Tenerife, Spain
}

Received 27 November 2014 / Accepted 18 November 2015

\section{ABSTRACT}

\begin{abstract}
Context. Solar-analogue stars provide an excellent resource to study the Sun's evolution, i.e. the changes with time in stellar structure, activity, or rotation for solar-like stars. The unparalleled photometric data from the NASA space telescope Kepler allows us to study and characterise solar-like stars through asteroseismology.

Aims. We aim to spectroscopically investigate the fundamental parameter and chromospheric activity of solar analogues and twins, based on observations obtained with the HERMES spectrograph and combine them with asteroseismology. Therefore, we need to build a solar atlas for the spectrograph, to provide accurate calibrations of the spectroscopically determined abundances of solar- and late-type stars observed with this instrument and thus perform differential spectroscopic comparisons.

Methods. We acquire high-resolution and high signal-to-noise $(\mathrm{S} / \mathrm{N})$ spectroscopy to construct three solar reference spectra by observing the reflected light of the asteroids Vesta and Victoria and the jovian moon Europa $(100 \lesssim S / N \lesssim 450)$ with the HERMES spectrograph. We then observe the Kepler solar analogue KIC $3241581(S / N \sim 170)$. For this star, the fundamental spectral parameters are extracted using a differential analysis. Sufficient $\mathrm{S} / \mathrm{N}$ in the near ultraviolet allows us to investigate the chromospheric magnetic activity in both objects.

Results. We constructed three solar spectrum atlases from 385 to $900 \mathrm{~nm}$, obtained with the HERMES spectrograph from observations of two bright asteroids and a jovian moon. A comparison between our solar spectra atlas to the Kurucz and HARPS solar spectrum shows an excellent agreement. KIC 3241581 was found to be a long-periodic binary system. The fundamental parameter for the stellar primary component are $T_{\text {eff }}=5689 \pm 11 \mathrm{~K}, \log g=4.385 \pm 0.005,[\mathrm{Fe} / \mathrm{H}]=+0.22 \pm 0.01$, being in agreement with the published global seismic values, which confirms its status as solar analogue. The chromospheric activity level is compatible with the solar magnetic activity observed during 2014 and 2015.

Conclusions. Our solar atlas is an essential tool for the analysis of solar-like stars and to characterise solar analogues and twins with HERMES. The differential analysis, using the presented solar atlas from HERMES observations allows us to obtain the fundamental parameters with very high accuracy. KIC 3241581 is a metal-rich solar analogue with a solar-like activity level in a binary system of unknown period.
\end{abstract}

Key words. stars: solar-type - stars: fundamental parameters - methods: observational - techniques: spectroscopic

\section{Introduction}

Solar-like stars and, in particular, solar analogues and twins provide a unique opportunity to understand our closest star, the Sun, in the context of stellar evolution. In the classical definition, provided by Cayrel de Strobel et al. (1981) and Cayrel de Strobel (1996; hereafter CdS96), solar twins are stars that are spectroscopically and photometrically identical to the Sun, while solar

* Based on observations made with the HERMES spectrograph mounted on the $1.2 \mathrm{~m}$ Mercator Telescope at the Spanish Observatorio del Roque de los Muchachos of the Instituto de Astrofísica de Canarias. $\star \star$ The solar atlases and the spectrum (FITS file) are only available at the CDS via anonymous ftp to

cdsarc.u-strasbg.fr (130.79.128.5) or via

http://cdsarc.u-strasbg.fr/viz-bin/qcat?]/A+A/589/A27 analogues are stars within $10 \%$ of the solar radius and mass, as well as having metallicities within $0.3 \mathrm{dex}$, but the definition allows for different ages.

The increasing number of known solar analogues allows us to study similar stars to the Sun but with differences in some of its properties, such as stellar rotation, chemical composition, or surface magnetic activity (e.g. García et al. 2014a; Baumann et al. 2010; Schrijver \& Zwaan 2008). Moreover, it is possible to place the Sun in its evolutionary context, as well as studying aspects of habitability through direct observations of individual targets or following statistical approaches (e.g. Unterborn et al. 2015).

Recently, asteroseismic quantities have been included in the definition of solar analogues. However, the precise observation of solar-like oscillators is challenging, as the pulsations 
exhibit amplitudes of a few parts per million in photometry or at the level of meters per second in Doppler velocity. Therefore, ground-based observations are only possible with high accuracy radial-velocity measurements (e.g. Aerts et al. 2010, and references therein). The advent of space telescopes, such as CoRoT (Baglin et al. 2006) and Kepler (Borucki et al. 2010), allows us to study larger datasets of solar-like oscillators. Prime examples are the characterisation of some solar analogues, such as 16 Cyg A\&B from Kepler observations (e.g. Metcalfe et al. 2012; do Nascimento et al. 2014; Davies et al. 2015) and CoRoT 102684698 (do Nascimento et al. 2013).

There are several convincing arguments for using asteroseismology to search for solar analogues and twins. The seismic key quantities of solar-like oscillations, the maximum oscillation power $v_{\max }$, and the separation between consecutive radial modes $\Delta v$ correspond to the surface gravity and the mean sound speed, and therefore allow us to derive the mass and radius of a solar-like oscillator with better than $10 \%$ precision, as shown by large sample studies for various evolutionary stages of single field stars (e.g. Chaplin et al. 2014; Kallinger et al. 2012), cluster members (e.g. Hekker et al. 2011; Miglio et al. 2012; Corsaro et al. 2012), and a comparison with dynamical masses from double-lined binary systems (e.g. Frandsen et al. 2013). A detailed analysis of the individual frequencies allows for an even more comprehensive study of solar-like stars and solar analogues, including a detailed comparison of their stellar structure (e.g. Metcalfe et al. 2012, 2014; Mathur et al. 2012; Silva Aguirre et al. 2015).

To unfold its full potential, seismology needs to be combined with additional parameters, taken from complimentary techniques. Lebreton \& Goupil (2014), as well as Chaplin et al. (2014), have demonstrated that very high accuracy is reached when seismology is combined with spectroscopically derived effective temperatures and abundances. For a subset of 90 solarlike oscillating stars, for which Bruntt et al. (2012) provide spectroscopic fundamental parameters, Chaplin et al. (2014) showed that, using the actual chemical abundances for each star, instead of assuming a mean abundance of $[\mathrm{Fe} / \mathrm{H}]=-0.2$ for the Kepler field, that the precision of seismic parameters for each single star increases. The combination of seismology with spectroscopically determined parameters, instead of photometric calibrations, improves the uncertainties by a factor of two, down to $\sim 5$ and $\sim 2 \%$ in mass and radius, respectively. However, obtaining precise spectroscopic values for space targets is challenging, since many of these targets are rather faint (e.g. from the Kepler mission) and high-resolution spectroscopy is lightdemanding but requires high signal-to-noise ratios $(S / N>100)$. In addition, detailed calibration is needed to achieve the highest precision possible. Using synthetic spectra for this purpose limits the accuracy in the derived chemical composition of stars by typical $\sim 0.1 \mathrm{dex}$, owing to inaccuracies in atomic and molecular data that enter calculations of the model atmospheres. This restraint in the analysis of the stellar spectrum can be overcome by comparing it to the solar spectrum, obtained with the same instrument and setup in close temporal proximity. With this observing strategy, both spectra suffer from similar systematics, such as imperfections in the wavelength calibration and hence, observations can be calibrated using the well-known parameters of the Sun.

To obtain the best spectroscopic calibrations for seismic studies and, in particular, for solar twins and analogues, efforts were spent on the construction of a calibrated solar reference spectrum, which was obtained with the HERMES instrument.
These reference spectra are available to the public through this publication.

Having built this reference solar spectrum, we apply it to the study of the Kepler target KIC 3241581. This star was selected because it provides a close match to the solar global seismic parameters from the $\sim 500$ Kepler stars that were analysed by Chaplin et al. (2014). KIC 3241581 also has a surfaceaveraged rotation period of around 26 days (García et al. 2014a), which implies that this star would be at around half of its mainsequence evolution, assuming general gyrochonology relations.

The analysis presented here provides a detailed spectroscopic study from high-resolution spectra, obtained with the HERMES spectrograph, to test how well this seismically selected solar analogue resembles the CdS96 criterion for solar analogue abundances, and to provide well-constrained fundamental parameters for further theoretical modelling.

Finally, we can also determine the level of stellar activity from spectroscopy. The level of chromospheric activity as well as the duration of the activity cycles are age dependent. Typically, the chromospheric activity is measured from the emission of the core of the CaII H\&K lines in the near ultraviolet, as established by Duncan et al. (1991, and references therein), from the data from an extensive observing program at the Mount Wilson Observatory (MWO). Through a set of newly observed targets from the list of Duncan et al. (1991), the $\mathcal{S}$-index for solar-like stars was calibrated for HERMES spectroscopy and applied to the solar analogue KIC 3241581.

\section{Observations}

The spectroscopic observations of the reflected solar light and KIC 3241581 ( $V=10.35 \mathrm{mag})$, which are presented in this paper were carried out with the High Efficiency and Resolution Mercator Échelle Spectrograph (HERMES, Raskin et al. 2011; Raskin 2011) mounted on the $1.2 \mathrm{~m}$ Mercator Telescope at the Observatorio del Roque de los Muchachos on La Palma, Canary Islands, Spain. The observations were obtained with the 2.5 arc seconds fibre for the high-resolution mode (HRF) with a resolving power of $R_{\text {HERMES }}=\lambda / \delta \lambda \simeq 85000$ (whereby $\lambda$ and $\Delta \lambda$ are the wavelength and the width per wavelength bin, respectively), covering a wavelength range of $385 \mathrm{~nm}$ to $900 \mathrm{~nm}$. The reference wavelength calibration was obtained from the combined light from a hollow-cathode Thorium-Argon and a Neon arc lamp (hereafter referred to as ThArNe).

For the acquisition of a solar references spectrum, observations taken from a point source are better suited than those taken from the sky. The latter suffers from scattering in the Earth atmosphere, which alters the measured spectrum depending on the angle between the observed spot in the sky and the Sun (Gray et al. 2000) and can modify the determined parameter by a few percent. Therefore, we followed the standard approach to obtain solar spectra from the light that is reflected from three different minor bodies in the solar system. For the asteroids 4 Vesta ( 7.4 mag) and 12 Victoria ( $9.4 \mathrm{mag})$, we obtained three spectra each in the night of July 30 to 31,2014 . This led to an accumulated integration time of one hour per object. The journal of observations and the obtained radial velocities are given in Table 1. Spectra of KIC 3241581 were acquired on those nights as well.

Over a timespan of 426 days, 20 spectra of KIC 3241581 with a total integration time of $8.1 \mathrm{hrs}$ were obtained, with the same setup than for the solar light (including the nights in which the solar spectra were observed). More details about the monitoring are presented in Sect. 6. 
P. G. Beck et al.: The HERMES solar atlas and the spectroscopic analysis of the seismic solar analogue KIC 3241581

Table 1. Journal of observations of the solar spectra, obtained from the reflected light on the bright asteroids 4 Vesta and 12 Victoria.

\begin{tabular}{lcccccc}
\hline \hline $\begin{array}{l}\text { Point } \\
\text { source }\end{array}$ & $\begin{array}{c}\text { Date } \\
\text { [Gregorian] }\end{array}$ & $\begin{array}{c}\text { Date } \\
\text { [HJD - 2456500.0] }\end{array}$ & $\begin{array}{c}\text { Exp. time } \\
{[\mathrm{s}]}\end{array}$ & $\begin{array}{c}\text { Airmass } \\
{[]}\end{array}$ & $\begin{array}{c}\text { BVC } \\
{\left[\mathrm{km} \mathrm{s}^{-1}\right]}\end{array}$ & $\begin{array}{c}\text { RV } \\
{\left[\mathrm{km} \mathrm{s}^{-1}\right]}\end{array}$ \\
\hline Vesta & 2014, July 30 & 369.374174 & 1200 & 1.48 & -29.171 & $-10.710 \pm 0.001$ \\
Vesta & 2014, July 30 & 369.388641 & 1200 & 1.59 & -29.199 & $-10.718 \pm 0.001$ \\
Vesta & 2014, July 30 & 369.403109 & 1200 & 1.73 & -29.225 & $-10.725 \pm 0.001$ \\
\hline Victoria & 2014, July 31 & 369.580444 & 1200 & 1.20 & +19.638 & $12.405 \pm 0.001$ \\
Victoria & 2014, July 31 & 369.594914 & 1200 & 1.15 & +19.600 & $12.403 \pm 0.002$ \\
Victoria & 2014, July 31 & 369.609384 & 1200 & 1.11 & +19.561 & $12.405 \pm 0.001$ \\
\hline
\end{tabular}

Notes. The heliocentric Julian date (HJD), the dimensionless air mass, as well as the barycentric velocity correction (BVC) calculated by the pipeline, are given for the midpoint of the exposure time (Exp. time). The barycentric radial velocity (RV) and the corresponding uncertainty were determined from a weighted cross-correlation, as described in the text. All observations were taken during the night from July, 30 to July 31 , 2014.

Table 2. Observing sequences of the solar spectrum, obtained from the observations of the Jovian moon Europa.

\begin{tabular}{lcccccccc}
\hline \hline $\begin{array}{l}\text { Point } \\
\text { source }\end{array}$ & $\begin{array}{c}\text { Date } \\
\text { [Gregorian] }\end{array}$ & $\begin{array}{c}\text { Exp. time } \\
{[\mathrm{s}]}\end{array}$ & $N$ & & $\begin{array}{c}\text { Date } \\
\text { [HJD - 2456500.0] }\end{array}$ & $\begin{array}{c}\text { Airmass } \\
{[]}\end{array}$ & $\begin{array}{c}\text { BVC } \\
{\left[\mathrm{km} \mathrm{s}^{-1}\right]}\end{array}$ & $\begin{array}{c}\text { RV } \\
{\left[\mathrm{km} \mathrm{s}^{-1}\right]}\end{array}$ \\
\hline Europa & 2015, April 17 & 400 & 5 & start: & 630.442 & 1.15 & -28.713 & $-8.398 \pm 0.003$ \\
& & & & end: & 630.463 & 1.21 & -28.747 & $-9.344 \pm 0.003$ \\
\hline Europa & 2015, April 17 & \multirow{2}{*}{200} & \multirow{2}{*}{19} & start: & 630.467 & 1.24 & -28.758 & $-9.521 \pm 0.003$ \\
& & & & end: & 630.519 & 1.71 & -28.855 & $-11.831 \pm 0.003$ \\
\hline Europa & 2015, April 19 & \multirow{2}{*}{200} & \multirow{2}{*}{32} & start: & 632.419 & 1.10 & -28.921 & $14.435 \pm 0.003$ \\
& & & & end: & 632.514 & 1.72 & -29.103 & $18.029 \pm 0.003$ \\
\hline
\end{tabular}

Notes. The column $N$ reports the number of integrations taken in a sequence with a constant exposure time. The values of HJD, air mass and BVC (cf. Table 1) are reported at the beginning and end of each observing sequence.

To test the stability of the HERMES spectrograph and to acquire a very high $\mathrm{S} / \mathrm{N}$ solar reference, we obtained another set of spectra from the Jovian moon Europa, with a total integration time of $3.4 \mathrm{~h}$ in additional runs on two nights between April 17 and 21, 2015. As a result of the rather high radial velocity variation with respect to the asteroids, the total integration time of $4.3 \mathrm{~h}$ was divided into 56 individual exposures of 400 and $200 \mathrm{~s}$, to minimise smearing effects. Details for the observing sequences are listed in Table 2. All observations of solar light were carried out on moonless nights. The compilation and normalisation of the combined spectrum and the validation of the method are presented in Sect. 3 .

The raw spectra that we obtained were reduced and wavelength-calibrated with the current version of the HERMES data-reduction pipeline (Version 5, Raskin et al. 2011). During the reduction of the spectra with the instrument-specific reduction pipeline, the spectra are rebinned to a constant resolving power of $R=85000$. The combined one-dimensional spectrum has no wavelength gaps, except for two small sections between échelle orders in the infrared between 857.7 and $858.1 \mathrm{~nm}$, as well as 878.6 and $879.5 \mathrm{~nm}$.

For all individual spectra the radial velocities were obtained through a weighted cross-correlation of the wavelength range between 478 and $653 \mathrm{~nm}$ of each spectrum with a G2 template (Raskin et al. 2011; Raskin \& Van Winckel 2014). From the analysis of long-time spectroscopic series of red giants, it was recently shown by Beck et al. $(2014,2015)$ that, with this approach, HERMES is capable of an excellent precision, allowing us to study solar-like oscillations with amplitudes of a few meters-per-second.

\section{The solar spectrum}

The availability and accuracy of the atomic and molecular data that enters calculations of model atmospheres and synthetic spectra is limited. In addition, this sort of modelling usually involves a fair number of approximations, such as hydrostatic and radiative equilibrium, and local thermodynamical equilibrium. All these factors limit the accuracy in the derived chemical compositions of stars to $\sim 0.1$ dex, typically. However, differential studies involving stars that are similar with regard to their atmospheric parameters can achieve a much higher precision. As an example, differential studies for solar analogues or twins (e.g. Ramírez et al. 2009; Meléndez et al. 2014; Nissen 2015) are routinely delivering relative abundances that are good to $\sim 0.01-0.02$ dex.

The Sun is the only star for which we know the atmospheric parameters (effective temperature and surface gravity) to a very high accuracy: about $3 \mathrm{~K}$ for Teff and 0.0001 dex for $\operatorname{logg}$ (Stix 1991). Hence, a precise differential analysis of a solar-like star relative to the Sun can be immediately translated into an accurate analysis. As we show in the tests below, we can measure the effective temperature of a star like the Sun relative to the Sun with a precision of a few degrees. Likewise, we can constrain the surface gravity of this kind of star to an excellent precision. Therefore, we can determine values to a high degree of accuracy, thanks to our knowledge of the absolute values of these quantities for the Sun.

To be able to perform these studies for stellar targets as well, using the HERMES spectrograph, we also aimed to obtain solar reference spectra for this instrument and understand 

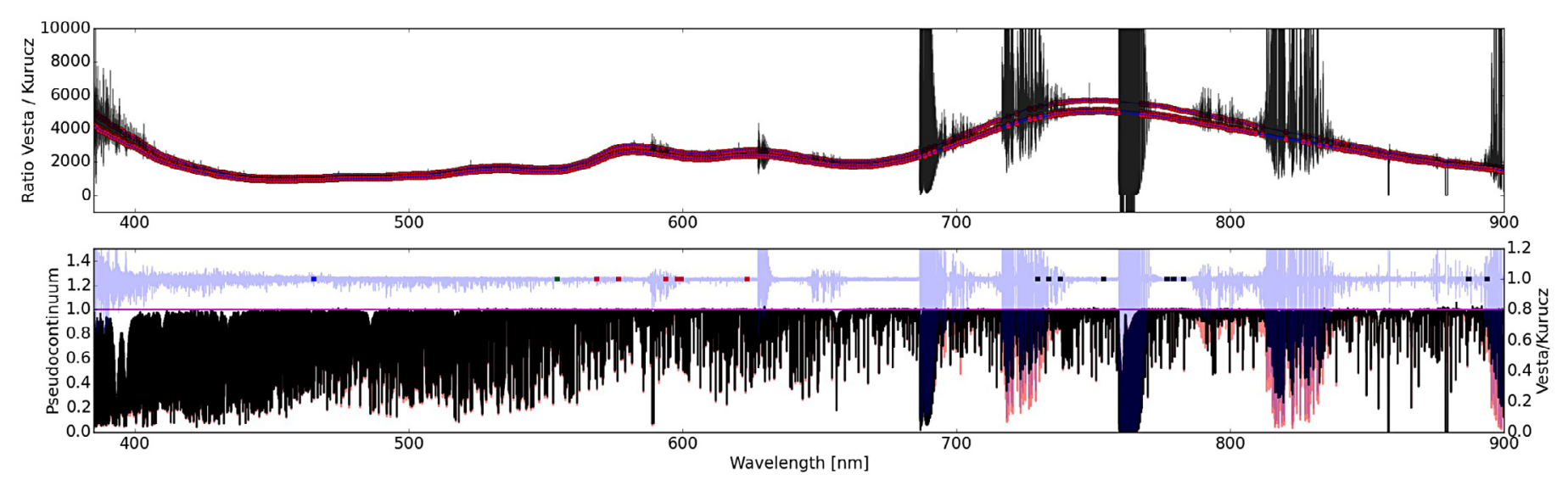

Fig. 1. Normalisation and compilation of the solar spectrum from observations of Vesta. The top panel shows the result of the division of the HERMES spectra by the Kurucz solar atlas for each individual exposure. Red dots indicate the supporting points for the cubic spline, depicted as a blue line. The regions of large deviations from the fit originate from contamination through telluric lines. The bottom panel depicts the resulting median spectrum obtained for the Vesta observations (in black) and the Kurucz (2005) spectrum (in red). The ratio between the spectra from Vesta and Kurucz are depicted as the blue line on the right hand y-axis. The regions used to determine the $\mathrm{S} / \mathrm{N}$ in Strömgren $b$, and $y$ as well as Johnson $R$ and $I$, are indicated in the bottom panel as blue, green, red, and black dots, respectively.

its characteristics. It will also be tested to see if the simultaneous acquisition of reference spectra is needed in the same night or if the reference spectra can be used over a longer time frame.

\subsection{Compilation of the solar atlas}

All reduced HERMES object spectra were corrected for the motion of the earth and the barycentric radial velocities that were measured from a cross-correlation are given in Table 1. To remove the trend that remains in the reduced one-dimensional spectra before compiling the combined spectrum, we divided the individual spectrum by the solar spectrum using the solar atlas of Kurucz (2005), appropriately smoothed as a reference. For more details on the reference spectrum, we refer to Sect. 3.3 in this paper. A cubic spline was fitted through 500 supporting points, that were equally spaced to map the long periodic variation in the ratio between the stellar and the reference spectra (Fig. 1, top panel). Each data point represents the median flux value in a window of $0.5 \mathrm{~nm}$. In the wavelength ranges where telluric lines are present, the spectra deviate strongly. For these regions, supporting points were placed manually to fit the continuum regions. Finally, the observed spectrum was divided by the cubic spline. By adopting this technique, we force the observed spectrum to follow the envelope of the pseudo continuum of the reference spectrum. This approach also corrects for the decrease of the intensity in the ultraviolet resulting from unresolved lines. The combined solar spectrum from each individual source (Fig. 1, bottom panel) was compiled by calculating the median flux from the individual spectra for each wavelength bin. We note that only spectra from the same source are merged.

The resulting spectrum from the reflected light from Europa is presented in Fig. 2. The location of telluric lines is visible through the strong outliers in the flux ratio between spectra of Vesta and Victoria (red line). Figure 3 illustrates the quality of the spectra for several regions, which is important for the spectroscopic analysis. In all observations, the width of the telluric lines was determined as being $\sim 2.5 \mathrm{~km} \mathrm{~s}^{-1}$ from the sigma of the Gaussian fit to the average telluric line profile.

Additionally, several telluric lines are found in emission. However, the intensity of this atmospheric night glow (e.g. Hilliard \& Shepherd 1966) hardly had any impact on the observed spectra.
Table 3. $\mathrm{S} / \mathrm{N}$ values in specific spectral pass bands.

\begin{tabular}{lcccc}
\hline \hline Wavelength & $N$ & 4 Vesta & 12 Victoria & Europa \\
\hline Acquisition year & & 2014 & 2014 & 2015 \\
\hline Strömgren $b$ & 4 & $210 \pm 20$ & $110 \pm 10$ & $470 \pm 200$ \\
Strömgren $y$ & 4 & $280 \pm 30$ & $130 \pm 10$ & $440 \pm 130$ \\
Johnson $R$ & 9 & $270 \pm 40$ & $150 \pm 20$ & $460 \pm 150$ \\
Johnson $I$ & 9 & $250 \pm 40$ & $130 \pm 20$ & $430 \pm 170$ \\
\hline
\end{tabular}

Notes. The reported $\mathrm{S} / \mathrm{N}$ represents the mean $\mathrm{S} / \mathrm{N}$ of $N$ continuum regions in the spectrum. The standard deviation from those subsets is given as uncertainty. The passband gives the location of the regions without stellar or telluric lines that were used to estimate the $\mathrm{S} / \mathrm{N}$ for the compiled solar median spectrum from the observations of the asteroids Vesta and Victoria and the jovian moon Europa.

Each median spectrum, e.g. as shown for Europa in Fig. 2, corresponds to a total integration time of $3600 \mathrm{~s}$, leading to a general $\mathrm{S} / \mathrm{N}$ that ranges between 110 and 470 for the various spectra. The $\mathrm{S} / \mathrm{N}$ was determined from regions, (ideally) without stellar or telluric absorption lines,

$$
S / N=\frac{1 .}{\operatorname{std}\left(F / F_{\text {mean }}\right)},
$$

whereby $\operatorname{std}\left(F / F_{\text {mean }}\right)$ is the standard deviation of the flux $F$ in the selected region, renormalised through the mean flux $F_{\text {mean }}$.

To find regions suited for the computation of the $\mathrm{S} / \mathrm{N}$, we compared the median spectra from HERMES observations to Kurucz's solar atlas, as well as to a synthetic spectrum of the Sun. This comparison showed that there are hardly any regions that are free from absorption lines, forcing us to adopt regions with minimal contamination too. Therefore, the reported $\mathrm{S} / \mathrm{N}$ is likely to be biased towards lower values through the result of the contamination from small lines. Table 3 lists the $\mathrm{S} / \mathrm{N}$ found for both median spectra, which was determined from such small snippets of continuum with respect to their corresponding classical photometric passbands. As this was calculated from several small sections of the spectrum, a mean $\mathrm{S} / \mathrm{N}$ value is reported, as well as the spread among the values. 
P. G. Beck et al.: The HERMES solar atlas and the spectroscopic analysis of the seismic solar analogue KIC 3241581

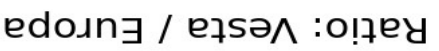

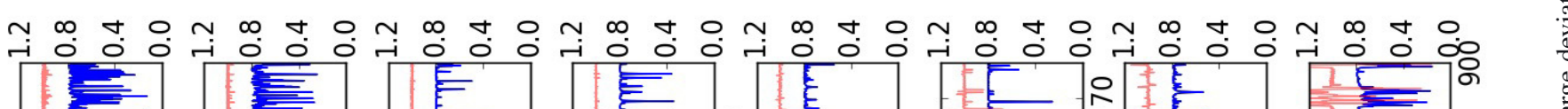

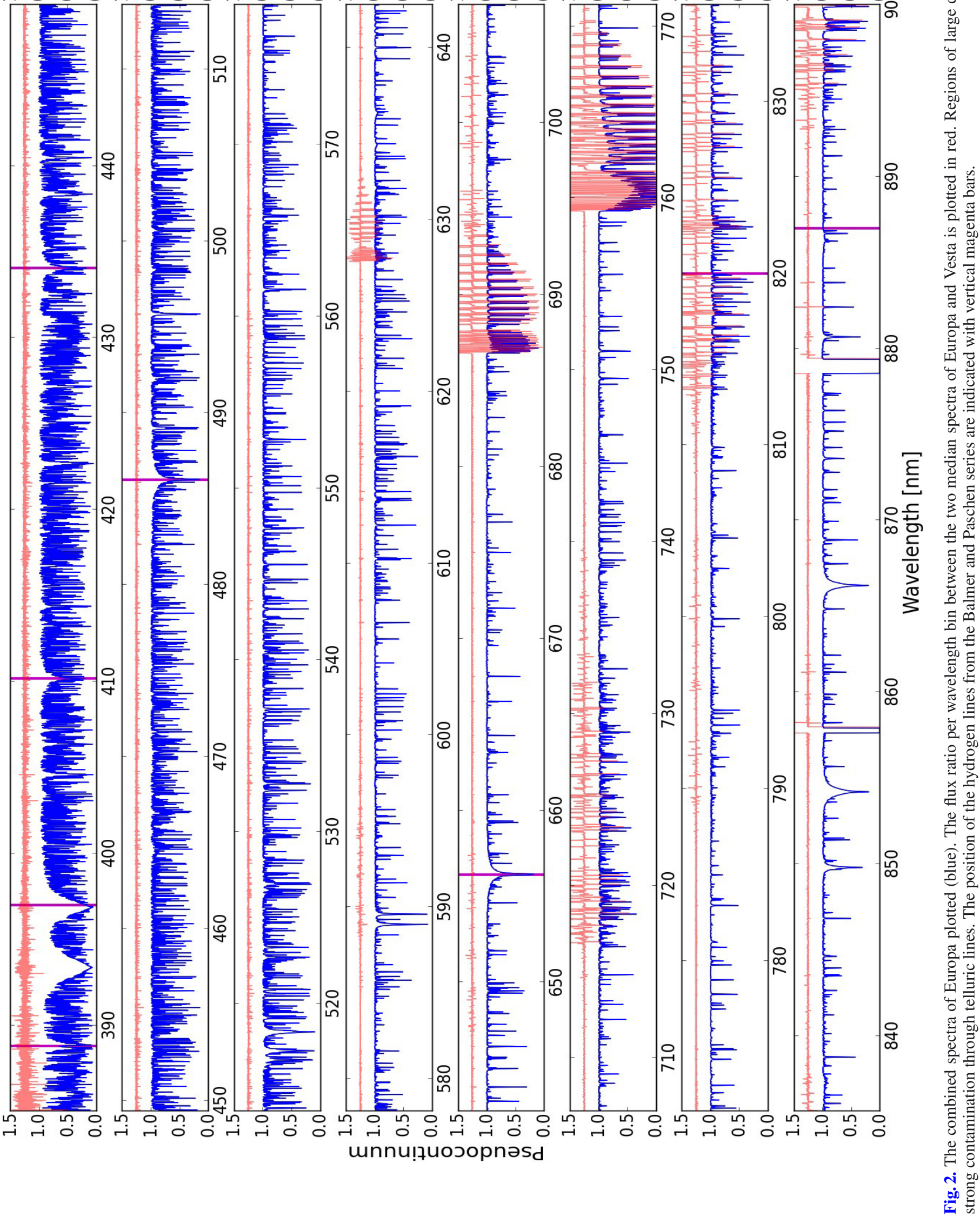



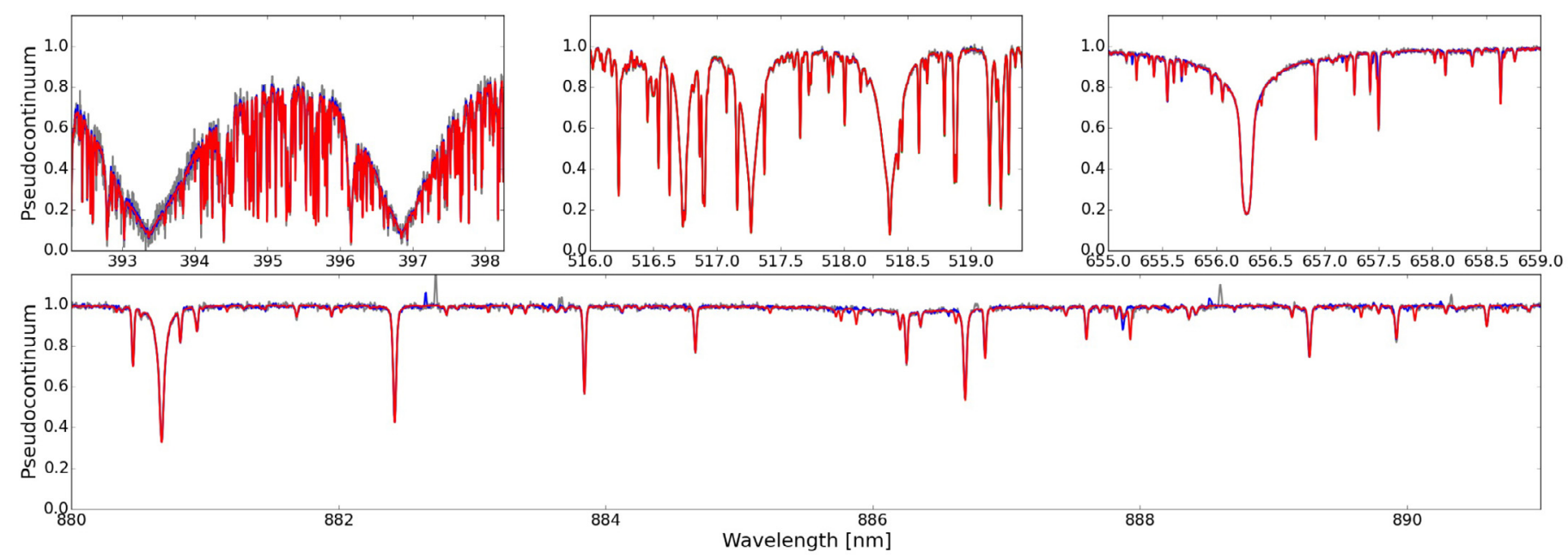

Fig. 3. Comparison of the HERMES spectra (blue and grey lines) with the smoothed Kurucz spectrum (red line) around the Ca H\&K lines (top left panel), the Mg-triplet (top centre panel) and the $\mathrm{H}_{\alpha}$ line (top right panel). The bottom panel shows the region in the infrared around the Paschen line at $887 \mathrm{~nm}$. The differences between the three spectra around $\mathrm{H}_{\alpha}$ and the Paschen line originate from the contamination casued by telluric lines. In the infrared, several weak telluric emission lines are also seen.

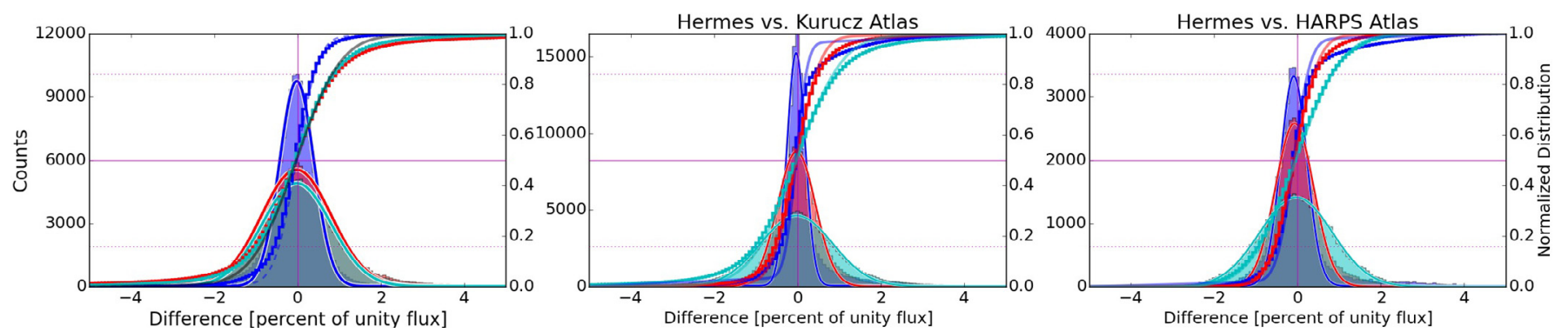

Fig. 4. Differential analysis of the spectra. The left panel shows the comparison between HERMES spectra of Europa, Vesta, and Victoria. The cyan histogram represents the distribution over the full spectral range, while the red histogram was calculated with the strongest telluric contribution excluded. The blue histogram indicates the distribution of the differences between the spectra of Vesta and Europa. The centre panel compares the two spectra from HERMES observations to the degraded Kurucz spectrum. In the right panel, the HERMES spectra are compared to the HARPS spectrum. In the centre and right panels, the blue, red, and cyan distributions depict the comparison of the reference spectra with the observations from Europa, Vesta, and Victoria, respectively. The same cleaning for telluric lines has been applied. The thin solid vertical and horizontal lines mark the theoretical centre of the distribution, while the dashed lines mark the borders of the $1 \sigma(68.27 \%)$ interval. Accumulated and normalised histograms are shown on the right axis. The thick grey line depicts the shape of the accumulated theoretical Gaussian distribution.

\subsection{Testing the consistency of HERMES solar spectra from different sources and times}

For a better understanding of the systematics contained in our observations, we compare the distributions of the difference in the flux per wavelength bin between the solar spectra obtained from HERMES and the solar atlases in literature.

Possible differences between the solar spectrum obtained from Europa, Vesta, and Victoria could be due to instrumental and atmospheric variations, or due to different characteristics of the asteroids, such as their albedo. The histograms of the differences of Europa and Victoria with respect to Vesta are shown in the left-hand panel of Fig. 4. Comparing the normalised, accumulated histograms (right-hand y-axis) with the accumulated theoretical Gaussian function, shows that the distributions of the differences bear a good resemblance to a Gaussian distribution. The peak of the Gaussian distribution (Table 4) is centred as expected at 0 within the resolution of the histogram $(\sim 0.08 \%)$.

Small differences in the raw data that originate from observations at different air masses (Tables 1 and 2) are removed by the normalisation to the pseudocontinuum. Since the observations of the three objects were separated in time, instrumental setup and the number of sunspots could, in principle, vary. No changes were made in the instrumental setup between the two observing runs. The international sunspot number, reported by the National Geophysical Data Center, NOAA ${ }^{1}$ for 2014 July, 30 and 31 were 88 and 95, respectively. For the observations of Europa, the sunspot number was in a similar range (2015, April 17-18: 85 and 100, respectively; April 20-21: 93). Those are rather small changes and do not strongly affect the result of the two spectra.

The two main systematic effects that contribute to the scatter are the presence of telluric lines and the different levels of $\mathrm{S} / \mathrm{N}$. The width of the distribution of the histograms in Fig. 4 is dominated by the lower $\mathrm{S} / \mathrm{N}$ in the spectrum of Victoria and also by the decreasing $\mathrm{S} / \mathrm{N}$ towards the blue part of both spectra (see Fig. 2). As a result of their position, depending on the stellar radial and barycentric velocity, telluric lines lead to a large number of outliers. Therefore, the histograms were calculated for the full spectral range (left-hand panel, cyan distribution) and excluding the regions that are most contaminated by telluric lines

\footnotetext{
1 http://www.ngdc.noaa.gov/stp/solar/ solardataservices.html
} 
Table 4. Centre and width of the Gaussian distributions of the residuals.

\begin{tabular}{lcccc}
\hline \hline & $\begin{array}{c}\text { Kurucz } \\
\text { [percent] }\end{array}$ & $\begin{array}{c}\text { HARPS } \\
\text { [percent] }\end{array}$ & $\begin{array}{c}\text { HERMES (full) } \\
\text { [percent] }\end{array}$ & $\begin{array}{c}\text { HERMES (cleaned) } \\
\text { [percent] }\end{array}$ \\
\hline Europa & $-0.03 \pm 0.25$ & $-0.08 \pm 0.30$ & - & $-0.02 \pm 0.43$ \\
Vesta & $-0.02 \pm 0.45$ & $-0.07 \pm 0.46$ & $-0.03 \pm 0.84$ & $-0.02 \pm 0.83$ \\
& & & \\
Victoria & $-0.02 \pm 0.86$ & $-0.03 \pm 0.87$ & & \\
Lit. Ref. & $+0.03 \pm 0.24$ &
\end{tabular}

Notes. The table reports the centre and width of the distribution of the residuals between different sources. The columns HERMES (full) and HERMES (cleaned) report on the residuals between two spectra obtained with HERMES for the spectra with and without the telluric lines, respectively. The row Lit. Ref. gives the distribution of the differences between the two reference atlases used in this work (cf. Fig. 5).

(red). Both distributions are centred on 0 within the histogram resolution.

Finally, we show the differences between Europa and Vesta in the left-hand panel of Fig. 4. For the simplicity of the diagram, only the spectra cleaned from heavy telluric contamination are compared. The ratio between the two spectra is also shown over the full range in red on the right- hand side of Fig. 2. Although nearly separated by $3 / 4$ of a year, the distribution of the differences is well centred on 0 , indicating that the quality of the spectrograph is stable and depends only on the $\mathrm{S} / \mathrm{N}$ obtained in the final spectra.

\subsection{Comparing solar HERMES spectra to solar atlases in the literature}

To compare our three solar median spectra from HERMES with published high fidelity solar atlases in the literature, we performed similar differential analyses with the revised FTS Kitt Peak Solar Flux Atlas of Kurucz (2005) and the laser calibrated solar atlas by Molaro et al. (2013), obtained from observations of the moon with the HARPS spectrograph.

The very high S/N spectrum of Kurucz (2005) was compiled from 50 Fourier transform spectroscopy (FTS) scans and extends beyond the blue and red borders of our spectra. The resolution of the spectrum ranges from $R=400000$ to 500000 and therefore had to be degraded to approximately fit the resolution $R_{\text {HERMES }}$. The atlas of Molaro et al. (2013) covers a wavelength range between 475 and $586 \mathrm{~nm}$ with a gap around $532 \mathrm{~nm}$. The original resolution of $R=115000$ was recalculated to $R_{\text {HERMES }}$. Since the published version of the spectrum is not normalised, we had to split the original file at $532 \mathrm{~nm}$ and normalise both échelle orders in the same way as described for our solar spectra by utilising the Kurucz spectrum as a template for the pseudocontinuum.

The distributions that compare the median HERMES spectra to the Kurucz and the renormalised HARPS atlases are shown in the centre and right panel of Fig. 4. All distributions are centred at zero (Table 4). The differences in the width of the distributions originates from the difference in $\mathrm{S} / \mathrm{N}$ among the three HERMES spectra (Table 3). Finally, Fig. 5 compares the distribution of the differences between the two literature atlases, smoothed to the spectral resolution of HERMES. Here the peak is centred on $0.03 \pm 0.24$.

\subsection{Data products}

By comparing the HERMES spectra to each other, as well as using the differential analysis with two published solar atlases, we

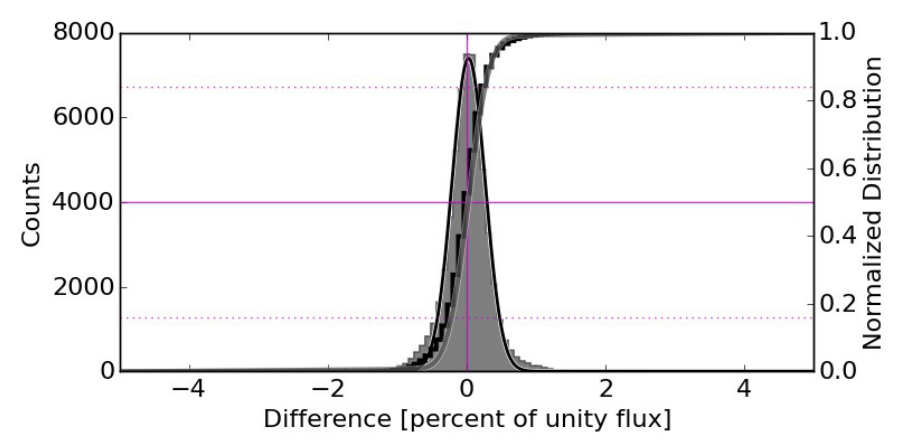

Fig. 5. Distribution of the differences between the Kurucz and the HARPS literature spectra.

conclude that the HERMES spectra are not significantly different. The level of $\mathrm{S} / \mathrm{N}$ found in both spectra is substantially higher than what is typically achieved in stellar spectra. Also the stability of the instrument over a longer timespan was confirmed, which indicates that the acquisition of solar calibration on the same night as the data is not needed to achieve high accuracy in the analysis.

We also find that our normalisation procedure works satisfactorily and delivers a pseudocontinuum that resembles the observations of Kurucz (2005). The only main source of the scatter and outliers are the differing levels of $\mathrm{S} / \mathrm{N}$ and the telluric lines, respectively.

To provide further users of the HERMES instrument with the possibility of calibrating their observations through the solar spectrum, the three compiled solar atlases obtained from Vesta and Victoria in 2014 and from Europa in 2015, and presented in this work, are available at the CDS. The full data package, also including raw and wavelength calibration data, will be available in an electronic archive on the webpage of the Mercator telescope $^{2}$ :

- The normalised median spectra for the solar spectrum (385-900 nm), obtained from Vesta, Victoria, and Europa, as described in the paper are provided.

- The individual, unnormalised integrations (377-900 nm) are available as one-dimensional FITS-files. These spectra are uncorrected for the radial and barycentric velocity. Several ThArNe spectra are available to determine the instrumental broadening of HERMES.

- The synthetic solar spectrum, calculated for infinite spectral resolution. Also the line list to identify the chemical elements for the absorption lines is given.

2 http://www.mercator.iac.es/ 
Table 5. Fundamental parameters of the Sun and KIC 3241581.

\begin{tabular}{|c|c|c|c|c|c|}
\hline \multirow{2}{*}{ Parameter } & \multirow{2}{*}{$\begin{array}{l}\text { Solar reference } \\
\text { Kurucz (2005) }\end{array}$} & \multicolumn{3}{|c|}{ HERMES Solar spectrum from source } & \multirow{2}{*}{$\begin{array}{c}\text { Solar analogue } \\
\text { KIC } 3241581\end{array}$} \\
\hline & & Europa & Vesta & Victoria & \\
\hline$T_{\text {eff }}[\mathrm{K}]$ & $5767 \pm 9$ & $5788 \pm 3$ & $5774 \pm 3$ & $5769 \pm 4$ & $5689 \pm 3$ \\
\hline $\log g[\operatorname{dex}]$ & $4.452 \pm 0.005$ & $4.453 \pm 0.006$ & $4.432 \pm 0.006$ & $4.426 \pm 0.005$ & $4.385 \pm 0.005$ \\
\hline$[\mathrm{Fe} / \mathrm{H}][\mathrm{dex}]$ & $0.000 \pm 0.002$ & $0.002 \pm 0.002$ & $-0.001 \pm 0.002$ & $0.000 \pm 0.002$ & $0.220 \pm 0.002$ \\
\hline
\end{tabular}

Notes. The effective temperature $T_{\text {eff }}$, projected surface velocity $v \sin i$, the surface gravity $\log g$, and the iron abundance $[\mathrm{Fe} / \mathrm{H}]$. The solar reference values for those parameters of the Sun are derived from Kurucz (2005). The deviation to the literature values of a given parameter, derived from the spectra atlases of Europa, Vesta and, Victoria, as well as the uncertainty of the measurement, are given. In the last column, the fundamental parameter of the seismic solar analogue KIC 3241581 are given. The error ranges reflect the internal uncertainties of the method and are discussed in the text.

- A selection of figures for teaching and public outreach will be presented on this page following the example of Beck (2012).

In addition to the three data sets of Vesta, Victoria, and Europa that already exist, new solar atlases from the ongoing monitoring of the mentioned targets, as well as additional minor bodies (such as Ceres), will be added to the database to provide reliable reference spectra for a variety of epochs. This growing sample of objects provides a selection of prominent celestial calibration targets and at least one of the objects will be visible throughout the year, which will allow us to compare new observations to the archived data.

We aim to make frequent reobservations of these targets during our yearly campaigns to further test the stability of the instrument. For the intermediate future, there are plans by the HERMES team to further maximise the flux efficiency of the fibre link between the telescope and the HERMES spectrograph and to optimise the wavelength calibration over the full wavelength range through instrumental updates. New calibrated spectral references atlases will be provided for the revised instrumental setup. This kind of archive will provide observers at, or users of, the HERMES data archive with a well-calibrated solar spectrum, taken in close temporal proximity and identical instrumental set-up in a few years. A consistent sets of standard-spectra will also allow us to compare the progress in the optimisation of the instrument.

The choice of standard spectral reference spectra is not limited only to the solar case. The optimal results are found if the star from which the "golden standard" spectrum originates from is as close as possible in mass, temperature, and radius to the target star. Such templates for a differential analysis would also allow an easier extraction of the activity tracers in late-type stars.

We therefore aim to extend the archive beyond mainsequence, solar-analogue stars, to, for example, $\gamma$ Doradus stars (e.g. Tkachenko et al. 2013) or stars in various phases of the red giant phase. Prime examples would be Arcturus (K0III) or the well described bright red giant in the wide binary system of $\theta^{1}$ Tau (=vb77 Tau, K0III), which is also a member of the very well studied Hyades cluster (Perryman et al. 1998; de Bruijne et al. 2001). This red giant has been characterised in detail through spectroscopic disentangling techniques from HERMES observations and seismic diagnostics from radial velocity time series (Beck et al., in prep.; 2015, respectively). This kind of reference spectra will also ease the process of normalisation and could serve as instrument-specific cross-correlation templates for these stars.

\section{The Sun as a reference}

To characterise the uncertainties that arise from using solar spectra as a reference for calibrating the spectroscopic libraries or in a differential analysis, we tested what the uncertainties are from using these spectra in a Sun-as-star experiment. The values of the Sun-as-star experiment (Table 5) were based on our solar spectra from Europa, Vesta, and Victoria in the wavelength range from 509 to $522 \mathrm{~nm}$, which includes the Mg I Triplet at $517 \mathrm{~nm}$. In addition, we also experimented with the Kurucz (2005) solar atlas, smoothed to the resolving power of the HERMES observations $(R=85000)$.

In our analysis approach, we compare the obtained spectra to a grid of synthetic spectra, with the three free parameters: $T_{\text {eff }}, \log g$ and $[\mathrm{Fe} / \mathrm{H}]$, with a fixed micro $=1.5 \mathrm{~km} \mathrm{~s}^{-1}$, ignoring macro turbulence. The analysis is carried out using FERRE (Allende Prieto et al. 2006) ${ }^{3}$. In the first step, we interpolated in the grid of synthetic spectra to obtain a model appropriate for the solar parameters $\left(T_{\text {eff }}=5777 \mathrm{~K}, \log g=4.437\right.$ and $[\mathrm{Fe} / \mathrm{H}]=0.00)$. We then modify our grid of models, multiplying all the model fluxes by the ratio of the observed and interpolated fluxes for the Sun. The resulting grid of models is therefore "forced" to match the solar observations, the median of HERMES fluxes for Europa, Vesta, and Victoria, at the solar parameters. The optimal solutions were found with FERRE using the UOBYQA algorithm (Powell 2000) and by performing a Bezier quadratic interpolation on the model grid. Our estimated formal uncertainties for both the Sun and KIC 3241581 compare favourably with those reported by Nissen (2015) for a set of solar analogues and a differential analysis relative to the Sun that is based on equivalent widths $\left(6 \mathrm{~K}\right.$ in $T_{\text {eff }}, 0.01$ in $\log g$. The results for the fundamental parameters and the $[\mathrm{Fe} / \mathrm{H}]$ abundance from the solar spectra in this Sun-as-star experiment are listed in Table 5, where the uncertainties correspond to $1 \sigma$ random errors derived by inverting the curvature matrix. Naturally, we find a good agreement with values in the literature and the uncertainties are dominated by the differing values of $\mathrm{S} / \mathrm{N}$ of each combined solar spectrum.

This approach allows us to carry out a differential analysis of the KIC 3241581 relative to the Sun. In addition to the internal uncertainties provided by FERRE, we 'empirically' computed our uncertainties from the dispersion found for the parameters of KIC 3241581 and the Sun, for which we have one and four spectra (Vesta, Victoria, Europa, and the Kurucz et al. solar atlas) available, respectively. We note that the intrinsic uncertainties and those we find empirically are fairly similar, and the values are within $11 \mathrm{~K}$ for $T_{\text {eff }}, 0.002 \mathrm{dex}$ for $[\mathrm{Fe} / \mathrm{H}]$, and about $0.01 \mathrm{dex}$ for $\operatorname{logg}$ to the solar value. We therefore adopt

FERRE is available from hebe. as. utexas. edu/ferre 
Table 6. Comparison of the $\mathcal{S}$-index for solar-like MWO-calibrator stars.

\begin{tabular}{llcccc}
\hline \hline Object & \multirow{2}{*}{ Spectral type } & \multicolumn{2}{c}{$\mathcal{S}$-index } & Spec & $\begin{array}{c}\text { Residuals } \\
\mathcal{S}-\alpha \cdot \mathcal{S}_{\mathrm{H}}\end{array}$ \\
& & MWO & HERMES & & 0.003 \\
HD66171 & G2V & $0.188 \pm 0.012$ & 0.0083 & 1 & 0.002 \\
HD88737 & F9V & $0.233 \pm 0.006$ & 0.0102 & 1 & 0.002 \\
HD114710 & F9.5V & $0.200 \pm 0.005$ & $0.0088 \pm 0.0001$ & 2 & 0.000 \\
HD115383 & G0V & $0.313 \pm 0.014$ & $0.0136 \pm 0.0001$ & 3 & 0.000 \\
HD115043 & G2V & $0.317 \pm 0.027$ & $0.0138 \pm 0.0001$ & 2 & 0.001 \\
HD120136 & F6IV & $0.188 \pm 0.005$ & $0.0082 \pm 0.0003$ & 6 & 0.006 \\
HD146233 & G2Va & $0.174 \pm 0.010$ & $0.0078 \pm 0.0029$ & 12 & 1 \\
HD190771 & G2V & $0.334 \pm 0.028$ & 0.0148 & 1 & 0.006 \\
\hline
\end{tabular}

Notes. The identifier, spectral type, and the total number of observations of the calibrator stars in the HERMES archive are given. The $\mathcal{S}$-index was calculated from the combined median HERMES data spectrum, following the procedure described in Sect. 3. The values reported for the Mount Wilson Observations (MWO) was calculated from the mean and standard deviation of all tabulated observations by Duncan et al. (1991). The error bars for the HERMES observations are calculated from the dispersion between the $\mathcal{S}$ calculated for individual spectra. The residual between MWO and HERMES are given. ${ }^{\star}$ ) Also known as 18 Sco.

Table 7. Chromospheric activity index $\mathcal{S}$ for the Sun from observations of the reflected light.

\begin{tabular}{llccc}
\hline \hline \multirow{1}{*}{ Object } & \multicolumn{1}{c}{ Date } & \multicolumn{2}{c}{$\mathcal{S}$-index } & Sunspot \\
& \multicolumn{1}{c}{ [Gregorian] } & $\mathcal{S}_{\text {HERMES }}^{\text {original }}$ & $\mathcal{S}_{\text {HERMES }}^{\text {MWO }}$ & number \\
\hline Moon & 2009, August 04 & 0.0077 & 0.18 & 0 \\
Vesta & 2014, July 30 & 0.0083 & 0.19 & $88-100$ \\
Victoria & 2014, July 31 & 0.0080 & 0.18 & $88-100$ \\
Europa & 2015, April 17\&20 & 0.0083 & 0.19 & $85-93$ \\
\hline KIC 3241581 & 2014-2015 & 0.0077 & 0.18 & - \\
\hline
\end{tabular}

Notes. Object used as source of reflected sunlight and the date of observations. The instrumental, MWO-scaled $\mathcal{S}$-index are given and compared to the international sunspot number.

these values as more conservative uncertanties for the parameters. These tiny uncertainties demonstrate the potential of a differential analysis relative to the Sun. The small scatter derived for the multiple solar spectra supports the small error bars for the derived parameters. In Sect. 6 we demonstrate that, since the atmospheric parameters of the Sun are known extremely precisely, we are able to derive accurate parameters for KIC 3241581.

\section{The calibration of the $\mathcal{S}$-index for HERMES spectroscopy of solar-like stars}

To quantify the activity level in late-type stars, we also aimed to determine the $\mathcal{S}$-index, an activity indicator based on the chromospheric emission in the core of the CaII H\&K absorption line in the near-ultraviolet. These lines are also included in the HERMES spectra.

To monitor the chromospheric activity of stars and to compare it to the large database of observations in the literature, we utilised the formalism of the $\mathcal{S}$-index of the Mount Wilson Observatory (MWO, Duncan et al. 1991, and references therein) that quantifies the level of chromospheric activity in the CaII H \& $\mathrm{K}$ lines,

$\mathcal{S}_{\text {HERMES }}=\frac{N_{H}+N_{K}}{N_{R}+N_{V}}$.

In this formalism, $N_{H}$ and $N_{K}$ are the fluxes in the centres of the $\mathrm{H}$ and $\mathrm{K}$ lines, weighted by a triangular filter with a FWHM of 0.109 nmcentred on 396.847 and $393.368 \mathrm{~nm}$, respectively. This flux is compared with the continuum flux, the two spectral segments $N_{R}$ and $N_{V}$ of $2 \mathrm{~nm}$, which are centred on 390.107 and $400.107 \mathrm{~nm}$, respectively.

Originally designed for a photon-counting spectrograph using photomultipliers, the fluxes corresponded to counts in the photometric passbands after the classical corrections (dead-time correction and sky correction). In the modern one-dimensional spectra, the surface under the pseudocontinuum replaces the counts in the photometric filters. The dimensionless indicator is dependent on the spectral resolution, as well as the colour index and the metallicity of the stellar object, and needs to be scaled through the multiplicative constant $\alpha$ onto the original value system for each instrument by using calibrator stars from the list of Duncan et al. (1991),

$\mathcal{S}_{\mathrm{HERMES}}^{\mathrm{MWO}}=\alpha_{\mathrm{solar}} \cdot \mathcal{S}_{\mathrm{HERMES}}$

We therefore obtained data for a set of eight calibrator stars from the original catalogue of MWO during an observing run in March and July 2015, as well as by searching the HERMES data archive. For these standard stars, the mean value for $\mathcal{S}$ from the literature, as well as the measured $\mathcal{S}$-index from HERMES spectra is listed in Table 6. The errors for MWO reflect the dispersion among measurements, reported in the catalogue. By fitting a linear regression to these pairs of values, we find that for solar-like stars, $\alpha_{\text {solar }}=23 \pm 2$. The main source of uncertainty in this comparison is the varying activity level in those solar-like stars, modulated by activity cycles, whereby we compare observations that are likely at different phases. Therefore a scatter is expected. The linear fit that we find gives a good result and has residuals better than $2 \%$. 


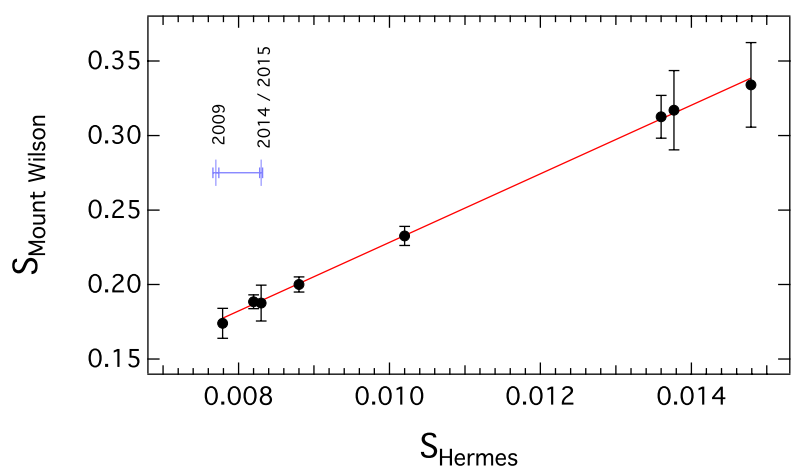

Fig. 6. Calibration of the $\mathcal{S}$-index by comparing the measured $\mathcal{S}$-index from HERMES with the average tabulated value from MWOobservations (cf. Table 6). For multiple observations, the vertical error bars reflect the scatter of those stars in the MWO catalogue, otherwise, the reported uncertainty was adopted. The red line depicts the linear correlation. For comparison, the instrumental $\mathcal{S}$-value of the Sun, measured in minimum phase of cycle 23 in August 2009 and in maximum phase of cycle 24 April 2015, on the horizontal axis is shown, reflecting the variation. The scatter of $\mathcal{S}$ within the time series of our observations of Europa is shown with the blue symbol in the upper left part of the diagram.

The $\mathcal{S}$-index found by our observations of the Sun via Vesta, Victoria, and Europa are reported in Table 7. These observations were conducted during the maximum of solar activity of the solar cycle 24 . To test the intrinsic scatter in high $\mathrm{S} / \mathrm{N}$ spectra, we calculated the dispersion of $\mathcal{S}$ in the 56 spectra from observations of Europa in April 2015 (Table 2), and found a typical scatter of \pm 0.0002 . To compare the Sun to the set of calibration stars, the measured solar-activity level (Table 7) is marked on the horizontal axis of Fig. 6. Naturally the $\mathcal{S}$-index of the Sun follows the solar activity cycle. In the archive of HERMES, we found unpublished observations of the solar spectrum, using the moon as a reflecting source from August, 4 2009. These observations were conducted during the recent minimum of solar activity. From those 12 spectra, we measure a mean value and dispersion of $\mathcal{S}=0.077 \pm 0.004$, respectively. Figure 6 compares the value from the solar minimum in 2009 to the $\mathcal{S}$-index of the recent (moderate) maximum in 2014-15, revealing how tiny the variation of $\mathcal{S}$ is amongst the solar cycle.

\section{Analysis of the seismic solar analogue KIC 3241581}

Seismic modelling, as well as evolutionary tracks, are very sensitive to chemical composition. It is therefore interesting to investigate how well a seismic solar analogue, selected from seismological indications such as KIC 3241581, does fulfil the third criterion of CdS96, which constrains the maximum deviation of the metal abundances to less than 0.3 dex. We obtained highresolution spectroscopy with the HERMES spectrograph. To test for binarity or variation in the tracers of chromospheric activity, we monitored this star with 20 observations spread over $\sim 1.5 \mathrm{yr}$.

The radial velocities obtained from the individual spectra, depicted in Fig. 7 (top panel) and listed in Table 8, reveal that KIC 3241581 is a binary system with a period substantially longer than $1.5 \mathrm{yr}$. The measured difference of currently more than $900 \mathrm{~m} / \mathrm{s}$ in radial velocity excludes a component with a substellar mass. A visual inspection of the spectra as well as the average line profiles from cross-correlation of the stellar

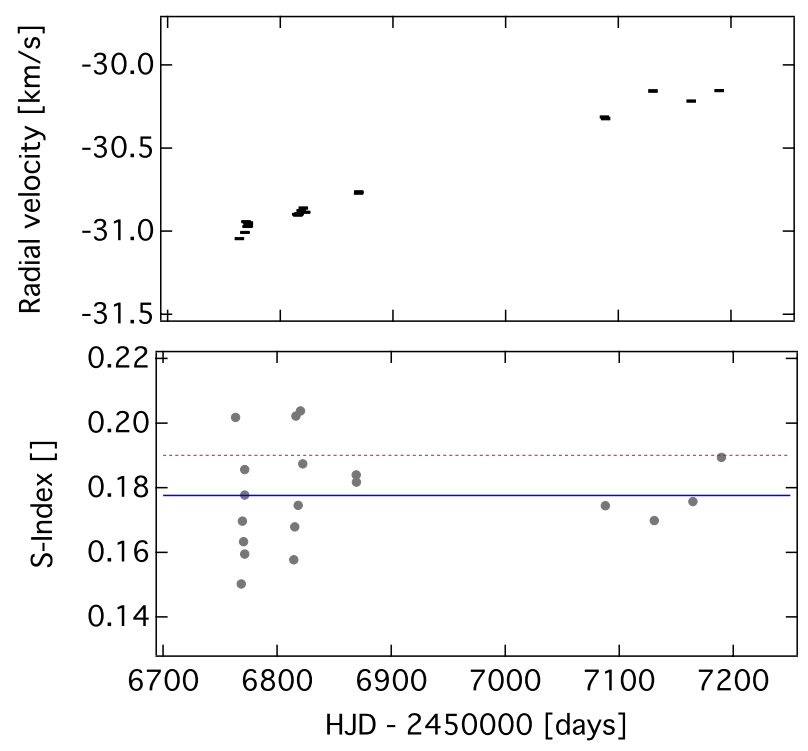

Fig. 7. Spectroscopic monitoring of KIC 3241581. Top panel: radial velocities of KIC 3241581. Bottom panel: the variation of the chromospheric activity indicator, $\mathcal{S}_{\text {HERMES }}^{\text {MWO }}$ of KIC 3241581 . The red line indicates the $\mathcal{S}$-index of the Sun from April 2015.

Table 8. Journal of observations and radial velocities of KIC 3241581.

\begin{tabular}{lcc}
\hline \hline HJD-2 456500 & RV $\left[\mathrm{km} \mathrm{s}^{-1}\right]$ & $\mathcal{S}$ \\
\hline 263.61191 & $-31.049 \pm 0.003$ & 0.202 \\
268.65827 & $-31.033 \pm 0.003$ & 0.151 \\
269.64710 & $-30.944 \pm 0.003$ & 0.170 \\
270.65539 & $-30.987 \pm 0.002$ & 0.163 \\
271.59569 & $-30.979 \pm 0.003$ & 0.178 \\
271.64209 & $-30.960 \pm 0.004$ & 0.160 \\
271.66548 & $-30.964 \pm 0.004$ & 0.186 \\
314.71648 & $-30.905 \pm 0.004$ & 0.158 \\
315.51440 & $-30.913 \pm 0.002$ & 0.168 \\
316.50856 & $-30.904 \pm 0.002$ & 0.202 \\
318.51228 & $-30.892 \pm 0.003$ & 0.174 \\
320.49913 & $-30.876 \pm 0.003$ & 0.206 \\
322.51981 & $-30.905 \pm 0.002$ & 0.188 \\
369.42749 & $-30.783 \pm 0.003$ & 0.184 \\
369.55871 & $-30.771 \pm 0.003$ & 0.182 \\
\hline 587.75066 & $-30.311 \pm 0.002$ & 0.175 \\
588.75505 & $-30.334 \pm 0.009$ & 0.080 \\
630.68656 & $-30.201 \pm 0.006$ & 0.168 \\
664.64064 & $-30.235 \pm 0.005$ & 0.175 \\
689.60817 & $-30.154 \pm 0.005$ & 0.190 \\
\hline
\end{tabular}

Notes. The heliocentric Julian date (HJD) is given for the midpoint of the exposure. The barycentric radial velocity (RV), the corresponding uncertainty as well as the value of the $\mathcal{S}$-index for the individual spectrum is reported.

spectrum with a G2 mask (see Sect. 2) did not show a spectroscopic signature of the secondary component in the individual composite spectra. Also no trace of a secondary is found in the power-spectral density. In the context of this paper, we refer to the primary of this system when using the KIC identifier. The photometric dilution that results from blending (Miglio et al. 2014) could be the cause for a lower $\mathrm{S} / \mathrm{N}$ in the oscillation power spectrum. Once the orbit and its elements are resolved, this object is an interesting candidate for the application of spectral disentangling techniques (e.g. FDBinary, Ilijic et al. 2004). 

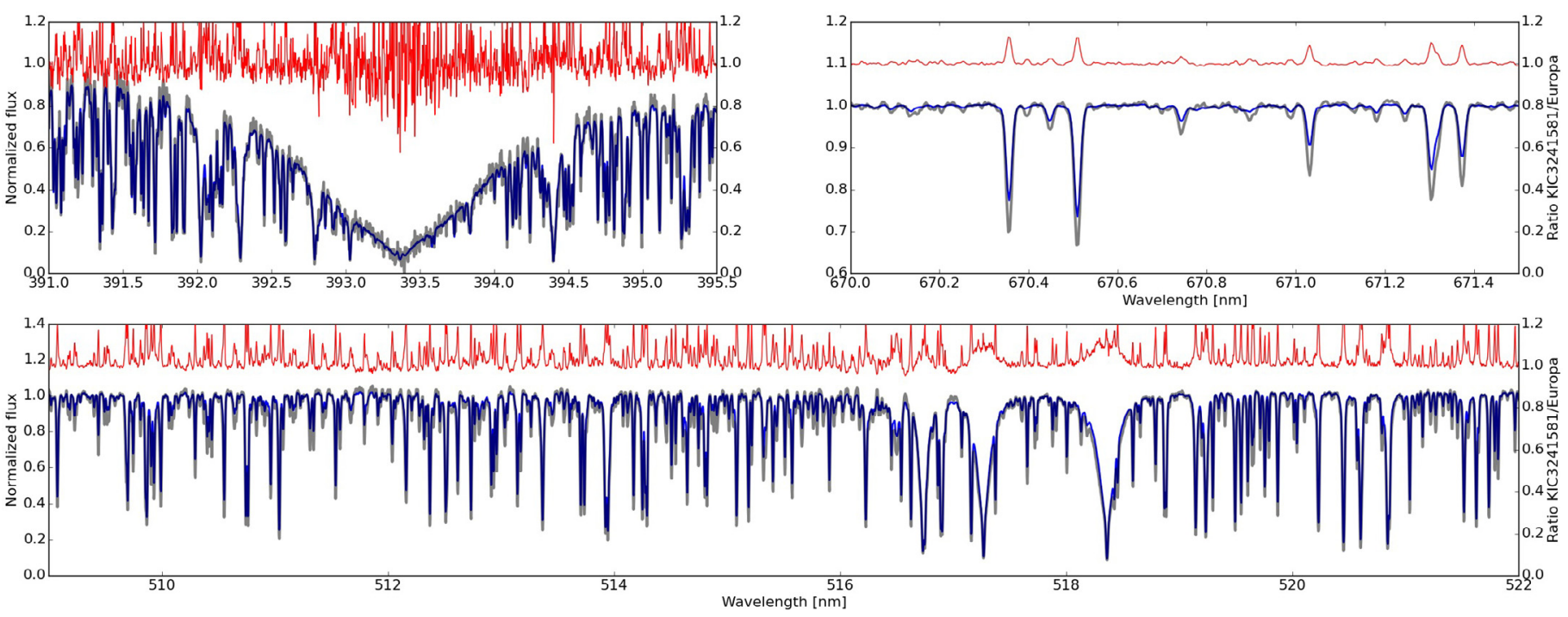

Fig. 8. Direct comparison of the spectra of the solar analogue KIC 3241581 (grey line) through the spectrum of Europa (blue). The value of the spectrum, normalised to the pseudocontinuum is given on the left $y$-axis. The red curve on top of the two spectra depicts the ratio between the flux per bin of KIC 3241581 and Vesta (right $y$-axis). The Ca K line, and the lithium doublet at $670.8 \mathrm{~nm}$ are shown in the left, and right panels, respectively, while the $\mathrm{Mg}$ triplet is depicted in the bottom panel.

Combining the 20 individual spectra obtained during the monitoring, and following the same procedure as described for the solar reference spectra, we obtain a median spectrum with a total integration time of $8.1 \mathrm{~h}$ and a $\mathrm{S} / \mathrm{N}$ of $\sim 170$ in Strömgren $y$. In Fig. 8, several important segments of the final spectrum of KIC 3241581 are shown and compared to the solar spectrum, which was obtained from Europa.

The fundamental spectroscopic parameters of the solar analogue were determined from the spectral range between 509 to $522 \mathrm{~nm}$, which surrounds the $\mathrm{Mg}$ triplet. Following the same analysis approach, as described for the Sun-as-a-star in Sect. 4, which uses the combined average of the three (reflected) solar spectra, the fundamental parameters of KIC 3241581 were determined to be $T_{\text {eff }}=5689 \pm 3, \log g=4.385 \pm 0.005$, and $[\mathrm{Fe} / \mathrm{H}]=0.220 \pm 0.002$. Yet, the retrieved $[\mathrm{Fe} / \mathrm{H}]$-abundance is still well within the range described by CdS96.

From the scaling relations of Chaplin et al. (2011), the seismic values reported by Garcia et al. (in prep.), $\nu_{\max }=2751 \mu \mathrm{Hz}$, $\Delta v=122.9 \pm 1.6 \mu \mathrm{Hz}$, and the effective temperature derived in this work reveal a star of $1.03 \pm 0.1 M_{\odot}, 1.08 \pm 0.1 R_{\odot}$ and $\log g=4.39 \pm 0.01$, which is well within the $10 \%$-range in mass and radius for solar analogues, defined by CdS96. The analysis of Garcia et al. (in prep.) has improved the seismic analysis of Chaplin et al. (2014) by improved data processing (García et al. 2014b) as well as the extraction of individual oscillation modes instead of using global seismic pipelines. Using the surface temperature and the radius determined from spectroscopy and asteroseismology, respectively, we find a stellar luminosity of $1.096 L_{\odot}$. The surface rotation rate remains stable with a period of $P_{\text {rot }} \simeq 26$ days (García et al. 2014a) and the value of photometric activity index, $\mathrm{Sph}=177.2 \mathrm{ppm}$ are close to the solar values of $P_{\text {rot }}^{\odot} \simeq 24.47$ days and $\mathrm{Sph}_{\odot}=166.2 \mathrm{ppm}$ (Salabert et al., in prep.).

The fundamental parameter, which is best constrained through global seismology, is the surface gravity, $\log g_{\star}$, since it is directly proportional to $v_{\max }$ of the star and can be constrained by more than than $2 \%$. It only further depends on the square root of the temperature ratio, $T_{\mathrm{eff}} / T_{\mathrm{eff}}^{\odot}$. Also the surface gravity is in agreement with the spectroscopy and seismology findings.
The average $\mathcal{S}$-index from the combined stellar spectrum of KIC 3241581, which is $\mathcal{S}=0.177$, is found slightly below the values found for the $\mathcal{S}$-value from our observations of the Sun (Table 7 and Fig. 7, bottom panel). Given the spread of the variations of the solar activity, KIC 3241581 shows a typical solar activity level. This kind of stellar activity, as well as the period of the surface rotation rate, indicate that the star is a mainsequence star (e.g. Skumanich 1972; Saar \& Brandenburg 1999). Recently, for solar-like stars observed with the Kepler satellite, Karoff et al. (2013) correlated ages that were derived from theoretical, seismic models with the CaH\&K excess flux and that provided a scaling relation for both quantities, which confirms the result of previous studies.

The lithium abundance of KIC 3241581 (Fig. 8, top right panel) was derived from the LiI resonance transition at $670.7 \mathrm{~nm}$. A synthetic spectrum was fitted to the HERMES spectrum, for the set of atmospheric parameters presented in Table 5 . $T_{\text {eff }}=5689 \mathrm{~K}, \log g=4.385 \mathrm{dex},[\mathrm{Fe} / \mathrm{H}]=+0.220$, and $\zeta=1.21 \mathrm{~km} \mathrm{~s}^{-1}$. Model atmospheres were interpolated in the Kurucz grid (Kurucz 1993) and the synthetic spectra were calculated with the MOOG routine (Sneden 1973). The synthesis of FeI lines in the $\lambda 670.7$ vicinity provides a low projected rotational velocity $v \sin i$. For the spectroscopic atmospheric parameters ${ }^{4}$, we derived $A_{\mathrm{Li}}=\log N(\mathrm{Li})=0.03$ dex. The value $A_{\mathrm{Li}} \sim 0.03$ is at the same scale and typical for a solar-analogues with a slightly higher mass than the solar value (Do Nascimento et al. 2009). For a typical one solar mass at the end of the PMS, the typical value of lithium abundance is around $A_{\mathrm{Li}} \sim 3.0$. The derived abundance value is therefore well in agreement with KIC 3241581 being on the main sequence.

\section{Discussion and conclusions}

To achieve the best accuracy possible for the spectroscopic analysis of solar-like stars and solar analogues and twins, we obtained solar reference spectra from light that has been reflected

4 In this work, we use the standard definitions: $[\mathrm{X} / \mathrm{Y}]=\log \left(N_{\mathrm{X}} / N_{\mathrm{Y}}\right)-$ $\log \left(N_{\mathrm{X}} / N_{\mathrm{Y}}\right)_{\odot}$, and $A_{\mathrm{X}}=\log \left(N_{\mathrm{X}} / N_{\mathrm{H}}\right)+12$, where $N_{\mathrm{X}}$ is the number density of element $\mathrm{X}$ in the stellar photosphere. 
from three minor bodies in the solar system, 4 Vesta, 12 Victoria, and Europa. The data products were validated through a direct comparison with two other solar atlases from the literature, as well on the basis of the derived fundamental parameters. An excellent agreement with found in both approaches. From the observation of solar-like stars, we calibrated the $\mathcal{S}$-index, a tracer for the chromospheric magnetic activity level as described by Duncan et al. (1991, and references therein) for the HERMES spectroscopy. Following the procedure for the compilation of the median spectrum, which is outlined in the paper, new solar reference catalogues will be produced to have recent solar spectra in close proximity to the ongoing monitoring in our solar analogue observing programme.

The solar analogue KIC 3241581 was identified from its close match of the global seismic quantities, $v_{\max }$ and $\Delta v$, and the surface rotation rate was derived from photometric time series collected with the Kepler space telescope. Using scaling relations, the star is found to be slightly more massive than the Sun, but is well within the boundaries of the definition of Cayrel de Strobel (1996, and references therein).

For more than $1.5 \mathrm{yr}$, KIC 3241581 has been monitored in high-resolution observing mode of the HERMES spectrograph, revealing that the star is the primary of a long periodic binary system. The spectroscopic fundamental parameters, as well as the metal abundance, were derived from a differential analysis. Using the solar spectrum, obtained from observations of the Jovian moon, Europa, as a template for the differential analysis, we determined the fundamental parameters to be $T_{\text {eff }}=$ $5689 \pm 3 \mathrm{~K}, \log g=4.385 \pm 0.005,[\mathrm{Fe} / \mathrm{H}]=+0.220 \pm 0.002$ and found that the star is, indeed, within the metallicity interval given by the classical definition for solar analogues by CdS96. Further analysis showed that the chromospheric $\mathcal{S}$ activity index of KIC 3241581, as well as the lithium abundance, are in good agreement with being a main-sequence star.

In addition to the high-precision space photometry from a space mission such as Kepler and K2, ground-based spectroscopy at intermediate size telescopes such as Mercator or the currently built SoNG telescope network (Grundahl et al. 2011) provide crucial and independent information to understand the nature of the stars, such as abundances or the variability of the level of chromospheric activity. Having available a highprecision calibration tool, such as the high $\mathrm{S} / \mathrm{N}$ solar atlas, will help to better characterise these targets and derive accurate abundances as the input parameters for stellar models, as we have shown for KIC 3241581.

Acknowledgements. The observations are based on spectroscopy made with the Mercator Telescope, operated on the island of La Palma by the Flemish Community, at the Spanish Observatorio del Roque de los Muchachos of the Instituto de Astrofísica de Canarias. PGB acknowledges the ANR (Agence Nationale de la Recherche, France) program IDEE (n ANR-12-BS05-0008) "Interaction Des Étoiles et des Exoplanètes". TVR acknowledges financial support from the Fund for Scientific Research of Flanders (FWO), Belgium, under grant agreement G.0B69.13. AT is a Postdoctoral Fellow of the Fund for Scientific Research (FWO), Flanders, Belgium. E.C. is funded by the European Community's Seventh Framework Programme (FP7/2007/2013) under grant agreement No 312844 (SPACEINN). The research leading to these results received funding from the European Community's Seventh Framework Programme ([FP7/2007-2013]) under grant agreement No. 312844 (SPACEINN) and under grant agreement No. 269194 (IRSES/ASK). PGB also received funding from the CNES grants at CEA. The authors thank Dr. Antonio Garcia for providing us with the archived solar spectra from 2009. We thank the anonymous referee for constructive criticism which helped to improve the paper.

\section{References}

Aerts, C., Christensen-Dalsgaard, J., \& Kurtz, D. W. 2010, Asteroseismology, Springer Science+Business Media B.V.

Allende Prieto, C., Beers, T. C., Wilhelm, R., et al. 2006, ApJ, 636, 804

Baglin, A., Auvergne, M., Barge, P., et al. 2006, in ESA SP 1306, eds. M. Fridlund, A. Baglin, J. Lochard, \& L. Conroy, 33

Baumann, P., Ramírez, I., Meléndez, J., Asplund, M., \& Lind, K. 2010, A\&A, 519, A87

Beck, P. G. 2012, Sterne und Weltraum, 2, 102

Beck, P. G., Hambleton, K., Vos, J., et al. 2014, A\&A, 564, A36

Beck, P. G., Kambe, E., Hillen, M., et al. 2015, A\&A, 573, A138

Borucki, W. J., Koch, D., Basri, G., et al. 2010, Science, 327, 977

Bruntt, H., Basu, S., Smalley, B., et al. 2012, MNRAS, 423, 122

Cayrel de Strobel, G. 1996, A\&ARv, 7, 243

Cayrel de Strobel, G., Knowles, N., Hernandez, G., \& Bentolila, C. 1981, A\&A, 94,1

Chaplin, W. J., Kjeldsen, H., Christensen-Dalsgaard, J., et al. 2011, Science, 332, 213

Chaplin, W. J., Basu, S., Huber, D., et al. 2014, ApJS, 210, 1

Corsaro, E., Stello, D., Huber, D., et al. 2012, ApJ, 757, 190

Davies, G. R., Chaplin, W. J., Farr, W. M., et al. 2015, MNRAS, 446, 2959

de Bruijne, J. H. J., Hoogerwerf, R., \& de Zeeuw, P. T. 2001, A\&A, 367, 111

Do Nascimento, Jr., J. D., Castro, M., Meléndez, J., et al. 2009, A\&A, 501, 687

do Nascimento, Jr., J.-D., Takeda, Y., Meléndez, J., et al. 2013, ApJ, 771, L31

do Nascimento, Jr., J.-D., García, R. A., Mathur, S., et al. 2014, ApJ, 790, L23

Duncan, D. K., Vaughan, A. H., Wilson, O. C., et al. 1991, ApJS, 76, 383

Frandsen, S., Lehmann, H., Hekker, S., et al. 2013, A\&A, 556, A138

García, R. A., Ceillier, T., Salabert, D., et al. 2014a, A\&A, 572, A34

García, R. A., Mathur, S., Pires, S., et al. 2014b, A\&A, 568, A10

Gray, D. F., Tycner, C., \& Brown, K. 2000, PASP, 112, 328

Grundahl, F., Christensen-Dalsgaard, J., Gråe Jørgensen, U., et al. 2011, J. Phys. Conf. Ser., 271, 012083

Hekker, S., Basu, S., Stello, D., et al. 2011, A\&A, 530, A100

Hilliard, R. L., \& Shepherd, G. G. 1966, Planet. Space Sci., 14, 383

Ilijic, S., Hensberge, H., Pavlovski, K., \& Freyhammer, L. M. 2004, in Spectroscopically and Spatially Resolving the Components of the Close Binary Stars, eds. R. W. Hilditch, H. Hensberge, \& K. Pavlovski, ASP Conf. Ser., 318, 111

Kallinger, T., Hekker, S., Mosser, B., et al. 2012, A\&A, 541, A51

Karoff, C., Metcalfe, T. S., Chaplin, W. J., et al. 2013, MNRAS, 433, 3227

Kurucz, R. L. 1993, SYNTHE spectrum synthesis programs and line data, Kurucz CD-ROM 18 (Cambridge, Mass.: SAO)

Kurucz, R. L. 2005, Mem. Soc. Astron. It. Suppl., 8, 189

Lebreton, Y., \& Goupil, M. J. 2014, A\&A, 569, A21

Mathur, S., Metcalfe, T. S., Woitaszek, M., et al. 2012, ApJ, 749, 152

Meléndez, J., Ramírez, I., Karakas, A. I., et al. 2014, ApJ, 791, 14

Metcalfe, T. S., Chaplin, W. J., Appourchaux, T., et al. 2012, ApJ, 748, L10

Metcalfe, T. S., Creevey, O. L., Doğan, G., et al. 2014, ApJS, 214, 27

Miglio, A., Brogaard, K., Stello, D., et al. 2012, MNRAS, 419, 2077

Miglio, A., Chaplin, W. J., Farmer, R., et al. 2014, ApJ, 784, L3

Molaro, P., Esposito, M., Monai, S., et al. 2013, A\&A, 560, A61

Nissen, P. E. 2015, A\&A, 579, A52

Perryman, M. A. C., Brown, A. G. A., Lebreton, Y., et al. 1998, A\&A, 331, 81

Powell, M. J. D. 2000, Report DAMTP 2000/NA14

Ramírez, I., Meléndez, J., \& Asplund, M. 2009, A\&A, 508, L17

Raskin, G. 2011, Ph.D. Thesis, Institute of Astronomy, Katholieke Universiteit Leuven, Belgium

Raskin, G., \& Van Winckel, H. 2014, Astron. Nachr., 335, 32

Raskin, G., van Winckel, H., Hensberge, H., et al. 2011, A\&A, 526, A69

Saar, S. H., \& Brandenburg, A. 1999, ApJ, 524, 295

Schrijver, C. J., \& Zwaan, C. 2008, Solar and Stellar Magnetic Activity (Cambridge, UK: Cambridge University Press)

Silva Aguirre, V., Davies, G. R., Basu, S., et al. 2015, MNRAS, 452, 2127

Skumanich, A. 1972, ApJ, 171, 565

Sneden, C. A. 1973, Ph.D. Thesis, The Univerisity of Texas at Austin, 35, 28

Stix, M. 1991, The Sun. an Introduction, 390

Tkachenko, A., Aerts, C., Yakushechkin, A., et al. 2013, A\&A, 556, A52

Unterborn, C. T., Johnson, J. A., \& Panero, W. R. 2015, ApJ, 806, 139 